\title{
DESIGN AND TESTING OF A HIGHLY MOBILE INSECT-INSPIRED AUTONOMOUS ROBOT IN A BEACH ENVIRONMENT
}

\author{
A.S. BOXERBAUM ${ }^{1}$, R.J. BACHMANN ${ }^{1}$, R.D. QUINN ${ }^{1}$, R.M. HARKINS ${ }^{2}$, T. DUNBAR ${ }^{2}$, \\ S.C. BURGESS ${ }^{3}$ \& R. VAIDYANATHAN 3,4 \\ ${ }^{1}$ Department of Mechanical Engineering, Case Western Reserve University, Cleveland, Ohio, USA. \\ ${ }^{2}$ Department of Physics, Naval Postgraduate School, Monterey, California, USA. \\ ${ }^{3}$ Department of Mechanical Engineering, University of Bristol, Bristol, UK. \\ ${ }^{4}$ Department of Systems Engineering, Naval Postgraduate School, Monterey, California, USA.
}

\begin{abstract}
The capability of autonomous platforms to function on beaches and in the ocean surf-zone is critical for a wide range of military and civilian operations. Of particular importance is the ability to navigate autonomously through the rocky terrain, hard-packed moist sand, and loose dry sand characterizing this environment. The study of animal locomotion mechanisms can elucidate specific movement principles that can be applied to address these demands. In this work, we report the design, fabrication, control system development, simulation, and field testing of a biologically inspired autonomous robot for deployment and operation in an ocean beach environment. The robot successfully fuses a range of insect-inspired passive mechanisms with active autonomous control architectures to seamlessly adapt to and traverse through a range of challenging substrates. Field testing establishes the performance of the robot to navigate semi-rugged terrain in the surf-zone environment including soft to hard-packed sand, mild to medium inclines, and rocky terrain. Platform autonomy is shown to be effective for navigation and communication. The fusion of passive mechanisms and active control algorithms results in a robot with mobility comparable to a legged vehicle with a control system of comparable simplicity to a wheeled robot. Based on the success of this platform, we further introduce the design of a fully amphibious robot designed to extend its performance to completely undersea surroundings.
\end{abstract}

Keywords: advanced mobility, autonomous control, biologically inspired robotics, field robotics, legged vehicles, passive mechanisms, reduced actuation.

\section{INTRODUCTION}

The ability to employ autonomous robots in difficult terrain continues be a rich area for research. In particular, there has been significant interest in the development of robots capable of autonomous operation within beach and turbulent ocean surf-zone environments. Potential utilities for such a robot include mine clearing, terrain mapping, and scouting potential approach lanes for amphibious naval operations [1].

A number of research groups have constructed platforms with the eventual goal of facilitating operations of this nature. These have included wheeled and tracked variants such as the Foster-Miller Lemming [2], legged and crawling robots [3, 4], snake robots [5], and walking platforms such as AQUA [6] (based on the RHex [7] platform), which, with manual adjustments, may be transitioned from walking to swimming locomotion. To date, however, a rugged robot capable of robust autonomous locomotion has yet to be fully developed for operations such as beach mine detection and clearing. A major hindrance to this realization is the trade-off between complex mechanical designs facilitating mobility over several substrates versus the difficulty of controlling these structures with enough rigor for full autonomous operation.

Our on-going research has attempted to address this issue through the development of a hybrid wheel-leg platform (dubbed Whegs ${ }^{\mathrm{TM}}$ [8]) drawing inspiration from cockroach mobility principles. Common to many robots in this line is a single drive motor that powers six multi-spoked appendages

(C) 2009 WIT Press, www.witpress.com

ISSN: 1755-7437 (paper format), ISSN: 1755-7445 (online), http://journals.witpress.com

DOI: 10.2495/DNE-V4-N4-319-336 
called wheel-legs. Neighboring legs are offset by $60^{\circ}$, yielding a nominal tripod gait. The wheel-legs allow the robots to climb over larger obstacles than a vehicle with similarly sized wheels. Whegs ${ }^{\mathrm{TM}}$ robots have compliant mechanisms in all six of their axles. These mechanisms allow them to passively adapt their nominal tripod gait to irregular terrain. This compliance captures much of what the cockroach accomplishes with actions of its distal leg joints. Additionally, we have designed robots with the incorporation of a body flexion joint [1]. This actively controlled joint enables it to perform both of the above body flexion changes used by the cockroach, thereby improving its climbing ability.

\subsection{Scope of work}

Our previous laboratory demonstrations and limited field testing [1,9] illustrate intriguing potential for combining active and passive control mechanisms to achieve the autonomy and robustness necessary for operation in the rocky terrain, hard sand, and soft sand that characterize the surf-zone environment. We extend these findings to report the development of a robot capable of traversing all these terrains with no input from a human operator. The robot design, incorporation of passive mechanisms for traversing variable challenging terrain, the autonomous control system (hardware and software), and communication system are detailed. Results culminate with a full field demonstration of mobility and autonomy (waypoint navigation) for a facsimile mission on the beach executed at the US Naval Postgraduate School in Monterey, California, USA. Based on our results with the beach robot, this paper also proposes a novel design of an amphibious surf-zone robot. Several problems are addressed in the new design, including a waterproof body flexion joint and propulsive mechanisms for swimming.

\section{AUTONOMY AND TERRAIN ADAPTABILITY}

Vehicle operation over rugged terrain without operator intervention poses many unique challenges. The ocean surf-zone, in particular, presents a host of distinctive environmental issues. Paramount among these is the capacity to navigate various obstacles while transiting dramatically different terrain. For example, mobility tuned for soft sand is often not optimal, or even functional, for hard-packed sand or a rocky beach. A platform tuned to operate smoothly in wet hard-packed sand might be impaired in dry soft sand and would have trouble maintaining balance (e.g. 'high center') on a rocky beach unless endowed with the capacity to adapt to fluctuations in terrain. Another critical challenge is to provide reasonable path control, obstacle avoidance, and the capacity to forward communications and data while in autonomous mode. Figure 1 illustrates a potential operational scenario for a surf-zone robot with wheel-leg appendages moving over terrain typified by this region. The design for this robot is described in Section 5.

\subsection{Biologically inspired mobility solutions}

Cockroaches have remarkable locomotion abilities that provide a wealth of inspiration for robot design. This capacity can provide specific inspiration to address challenges of surf-zone operation. In studies of cockroach movement, we have noted the following [10] (as illustrated in Fig. 2):

- A cockroach has six legs that support and move its body.

- It typically walks and runs in an alternating tripod gait wherein the front and rear legs on one side of the body move in phase with the middle leg on the other side.

- Although the front legs swing head-high during normal walking so that many obstacles can be surmounted without significant gait change, the animal changes its gait when it encounters larger barriers. 


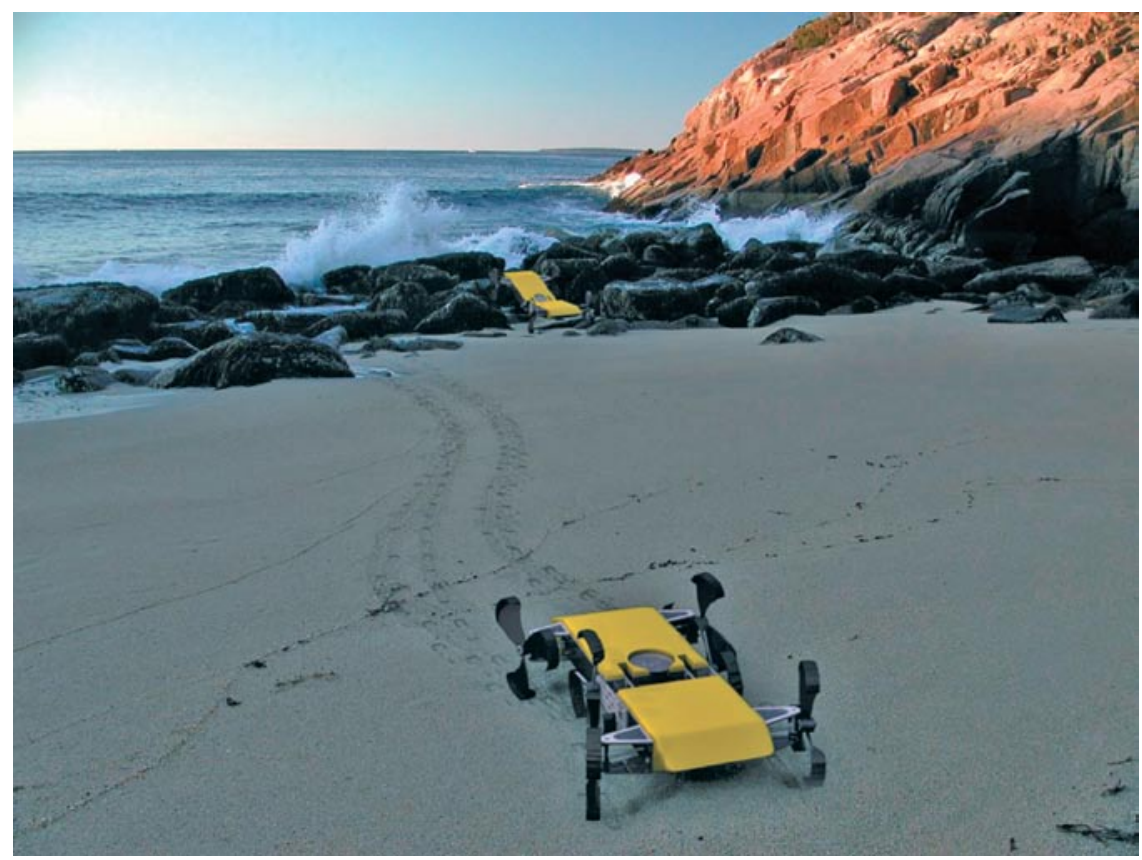

Figure 1: Image rendering of a robot moving from the water to the beach.
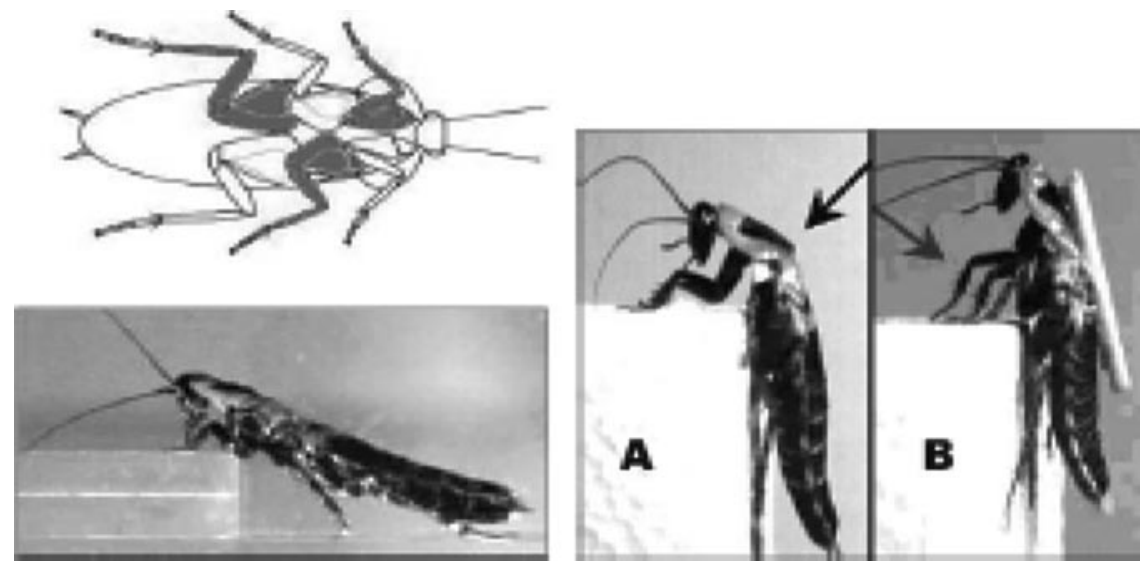

Figure 2: Cockroach climbing and tripod gait locomotion [13]: graphic of tripod gait (top left), change of posture to climb (lower left), body flexure while climbing (A), and difficulty in climbing with flexure restricted (B). Source: Photo courtesy of the Ritzmann Laboratory, Case Western Reserve University.

- The cockroach turns by generating asymmetrical motor activity in legs on either side of its body as they extend during stance [11].

- A cockroach enhances its climbing abilities by changing its body posture before and during a climb over an obstacle [12]. 
- It uses its middle legs to pitch its body up prior to climbing obstacles that are higher than its head, which enables its front legs to reach higher (Fig. 2, lower left).

- During a climb it uses flexion joints to bend the front half of its body down to avoid high centering (Fig. 2a and B).

We have developed a series of rugged all-terrain robotic vehicles dubbed Whegs ${ }^{\mathrm{TM}}$ [1] capable of fast running and climbing through the incorporation of all of the aforementioned biologically inspired mechanisms to navigate terrain. Common to robots in this line is a single drive motor that powers six multi-spoke appendages called wheel-legs. Neighboring legs are offset by $60^{\circ}$, yielding a nominal tripod gait. The spokes enable the capacity to climb over larger obstacles than a vehicle with similarly sized wheels. Whegs ${ }^{\mathrm{TM}}$ robots have compliant mechanisms in all six of their axles. These mechanisms allow them to passively adapt their tripod gait to irregular terrain. This compliance captures much of what the cockroach accomplishes with actions of its distal leg joints. Additionally, we have designed robots with an insect-like body flexion joint [14]. This actively controlled joint enables it to perform both of the above body flexion changes used by the cockroach, thereby improving its climbing ability.

\section{PROTOTYPE BEACH ROBOT}

Our Beach Whegs ${ }^{\mathrm{TM}}$ robot is designed with active and passive mechanisms for maximum mobility and terrain adaptability. The robot is propelled by a single motor to move in a cockroach-like tripod gait normally, but passively adapts its gait for mobility on different terrains. Through extensive field testing, we have isolated, tested, and integrated a range of subsystems designs to create a robot suited for autonomous operation. These innovations have resulted in a robust robot well suited to autonomous operation in the beach and other sandy/rocky environments.

\subsection{Mechanical design}

Features of this robot implemented to accommodate its size, payload, and capacity for operation on the beach include:

3.1.1 Structure and passive gait control mechanisms

Similar to other Whegs ${ }^{\mathrm{TM}}$ robots, a single drive motor powers multi-spoke appendages (called wheellegs) on the robot. Neighboring legs are offset by $60^{\circ}$, yielding a nominal cockroach-like tripod gait (Fig. 3). The spokes allow the robot to climb over larger obstacles than a vehicle with similarly sized wheels. The robot's mission of testing locomotion in sandy environments and demonstrating autonomy did not require a body flexion joint. Therefore, the body has a single segment with six wheel-legs.

The robot is equipped with compliant mechanisms in all six of its axles (Fig. 4). These mechanisms allow the robot to passively adapt its tripod gait to irregular terrain. This compliance captures much of what the cockroach accomplishes with its local neuromechanical system and greatly simplifies the robot's control system.

\subsubsection{Cable steering}

Whegs ${ }^{\mathrm{TM}}$ hexapod robots are steered by rotating their front and rear wheel-legs in opposite directions. Previous versions of Whegs ${ }^{\mathrm{TM}}$ use a hobby servo to drive a four-bar mechanism through a tie-rod. For the beach robot, we used a more powerful servomotor linked to the wheel-legs with a cable. This allows for a more compact design, consistent torque, and a larger range of motion, which provides a smaller turning radius. We have also found that the elasticity of the cable insulates the servo from harsh impacts the wheel-legs experience on rocky terrain. Several materials for the cable 


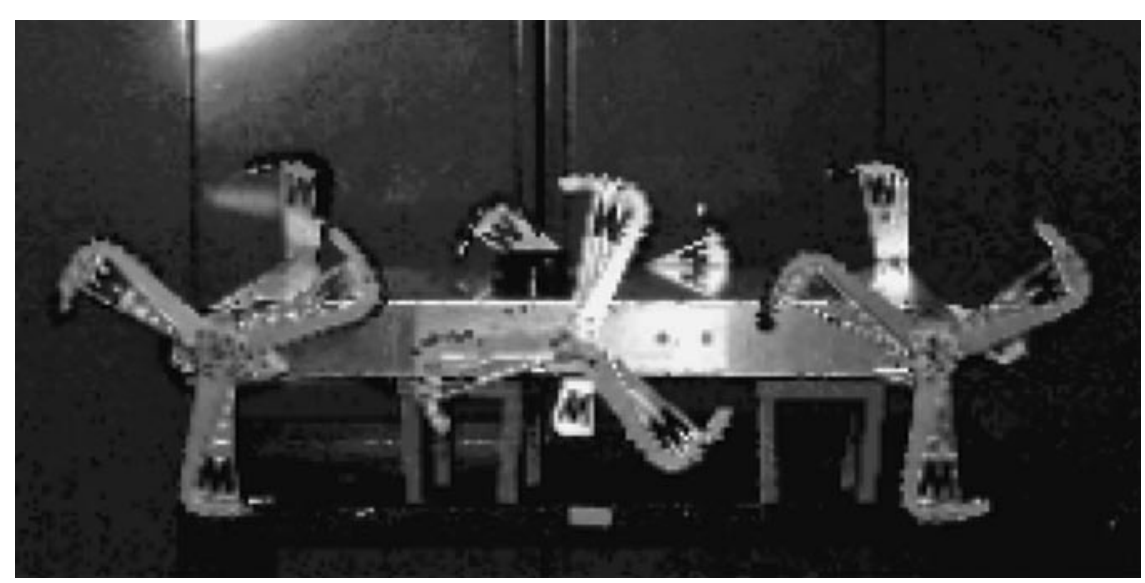

Figure 3: Prototype beach robot.

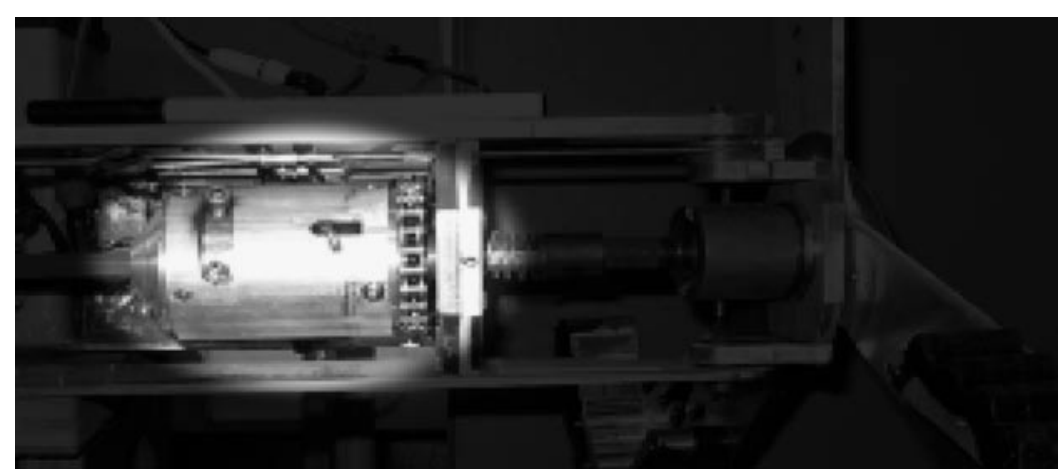

Figure 4: Passive compliance mechanism in an axle.

design were tested, including Spectra, Kevlar, and aircraft steel. The reoccurring problem was breakage of the cable due to fraying at the attachment points. Spectra did not fray when used with knots instead of screw plates; yet tension was inconsistent. Ultimately, a thicker steel cable with nylon coating was used with success.

\subsubsection{Foot design}

Two kinds of feet were tested for maximum terrain adaptability. One was designed for grassy fields and soil, consisting of an aluminum spike or claw offset from the spoke. This design performed well on those substrates. The spike penetrated the top layer of grass, giving it excellent traction on an otherwise slippery surface. Using these feet, the robot was able to climb up a $32^{\circ}$ slope hill. However, these feet were not suited for hard or sandy surfaces. There was no damping and the wheel radius was highly discontinuous. Furthermore, this foot had insufficient surface area to keep the robot from sinking into the sand. An alternate design was developed for more general use. It consisted of a one-inch wide aluminum and rubber foot with wave treads. This was tested in the Nevada 
desert and demonstrated excellent performance on rocks, gravel, packed dirt, and even indoor carpeting. This design also added the benefit of a 'zero-scrub' radius; i.e. the 1-inch wide foot was arranged to locate the center of the foot directly below the steering axis of rotation. This eliminates any moment about the steering axis caused by ground reaction forces. The design enabled stronger controllability, particularly at high speeds, and less wear on the steering servos.

\subsubsection{Partially enclosed body}

The field-tested robot design has a partially closed body with small openings along the seams. While not fully enclosed, the robot casing protected interior components from the environment. Future work will involve a fully sealed body for in-water operation.

\subsubsection{Heat}

The robot's sealed body inhibited heat dissipation, which became a problem in certain environments. The speed controllers and batteries were particularly vulnerable to issues with overheating. This was largely due to the low voltage motors we were using and the frequency of stall situations. Our 12-V motor has a stall current of $109 \mathrm{~A}$. The speed controllers are rated for that current for up to one second. However, stall conditions were hard to detect remotely, due to the compliance in the drive train. Furthermore, in the most extreme heat conditions of the Nevada desert, the ambient temperature may have contributed to poor speed controller performance. Future autonomous work will use higher voltage motors, in order to reduce the necessary current, and will address the need for stall detection in software, which could implement a controlled torque reduction. In this application, we installed a fan and several air filters to reduce box temperature. Furthermore, the interior was designed to separate heat-producing from heat-sensitive components, and custom heat sinks were built to transfer heat from the motor and speed controller to the outer aluminum wall of the robot.

3.1.6 Wide body and steering mechanism

The robot has a toe-to-toe width of $50 \mathrm{~cm}$ ( 20 inches) with a wheel-leg radius of $19 \mathrm{~cm}$ ( 7.5 inches). A wide body steering mechanism had to be designed to support robot turning. In rocky terrain, rocks small enough to pass between the legs had to be accounted for in design.

\subsubsection{Weight}

With Global Positioning System (GPS) equipment and all-terrain feet, the mass of Beach Whegs ${ }^{\mathrm{TM}}$ is $16.8 \mathrm{~kg}(37 \mathrm{lbs})$. When the drive electronics were running properly, giving full torque, this weight appeared to be acceptable. The robot could climb steep slopes and full-size stairs.

\subsection{Electronics hardware and controller design}

To realize autonomy, the robot was populated with various controllers and sensors. The heart of the platform was based on the Z-World Wildcat BL2000 microcontroller. The BL2000 uses a 22-MHz Rabbit processor that is programmed in $\mathrm{C}$ and comes with a ready function library and development environment.

A Garmin GPS unit was used for land-based positional awareness. An HMR2100 Honeywell digital magnetic compass with a built-in inclinometer was also installed on the vehicle. GPS and compass data update rates were on the order of $4 \mathrm{~Hz}$. A Netgear Access point was used for communication and data telemetry. A Crossbow accelerometer was utilized for inertial feedback.

For motor speed and direction control, a pulse width modulation (PWM) circuit was designed and constructed. For this circuit, a 555 timer chip was used to create a saw-tooth signal that was fed into the 
non-inverting side of an operational amplifier in comparator mode. Input from the digital-to-analog converter on the micro-controller was fed to the inverting input based on calculated control requirements. The result was a PWM signal for direction and speed control to the motors. The turn direction was implemented by the servos attached to the forward and aft wheel-legs. Figure 5 illustrates the output of the comparator as a function of the inputs.

\subsubsection{Communications}

The communications design for our platform was anchored on a UDP/IP stateless $802.11 \mathrm{~b} / \mathrm{g}$ protocol. A stateless protocol is preferred so that communication would not hang on a failed three-way handshake inherent in full state communications. Data to the robot included up to 10 waypoint latitude and longitude positions and an ability to drive the platform manually. The latter was a necessity for in-field experiments. A way to manually stop and maneuver the robot was essential to protect the robot during testing and integration. Figure 6 shows the communications ports as they relate to robot algorithms. Waypoint data is processed by the waypoint handling function, while manual control strings are passed directly to the PWM for direct control of the robot.

Outgoing data included real-time position and heading information and any error messages from the robot to the control station while in autonomous mode.

\subsubsection{Control}

The robot moves into autonomous mode when the control station sends waypoint route data to the platform on UDP port 4002. Figure 10 illustrates the functional and hierarchical relationships for the robot co-statements while autonomous. Co-statements are a Z-World implementation for cooperative multitasking (shared CPU time) between the control functions. As waypoint, compass, and GPS data are fed to the navigation function, plant signals are calculated and sent to the PWM which signals the motor-controllers to maneuver the robot.

Robot course and heading control is realized with PID control. The feedback loop is depicted in Fig. 7. Upon receipt of waypoint data, the robot calculates a desired course and range and figures

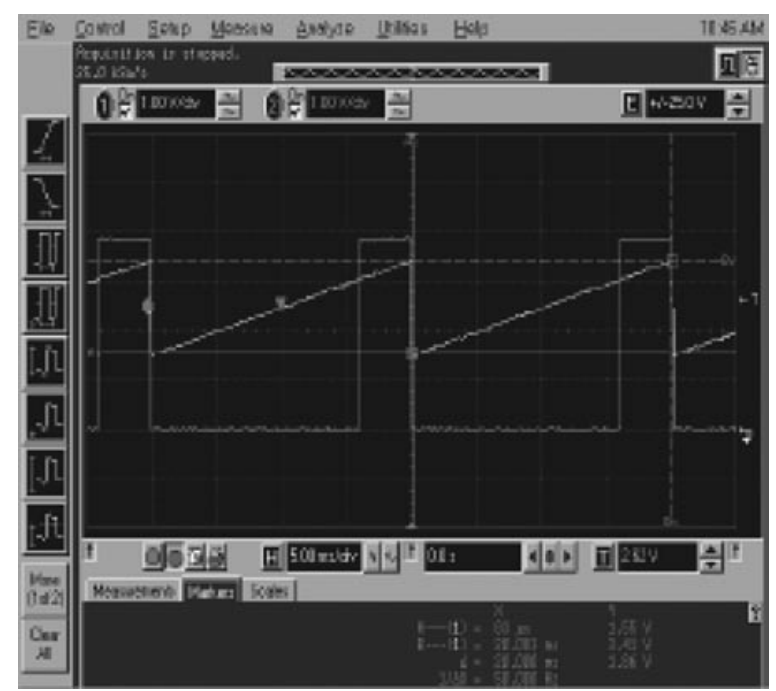

Figure 5: The PWM signal. 


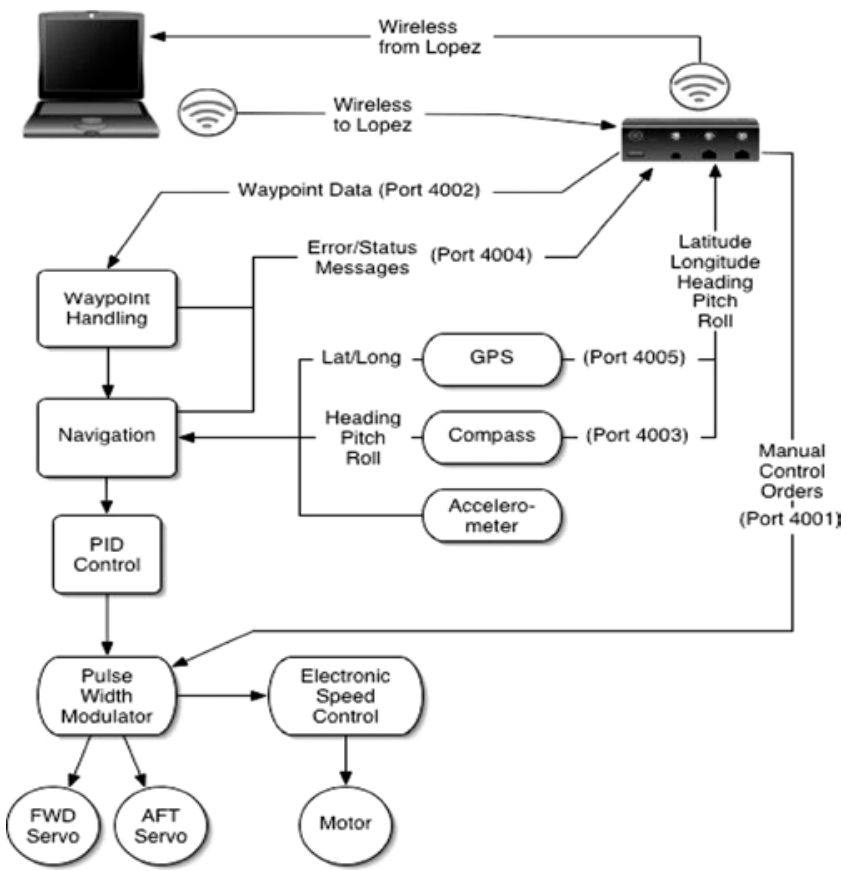

Figure 6: Robot communications architecture.

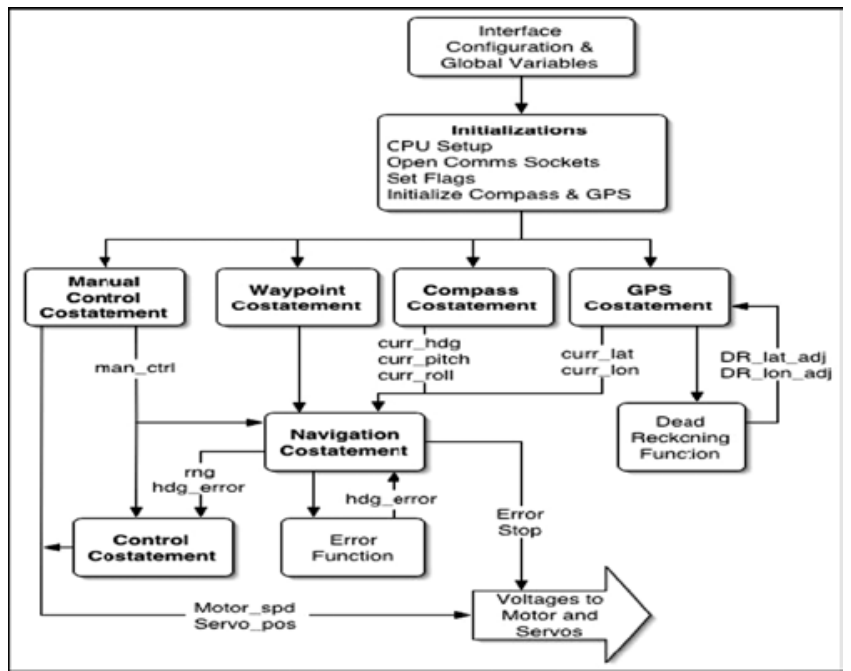

Figure 7: Robot autonomous control logic.

error, which is passed to the compensator. The compensator operates on the error and determines a signal (S) that is sent to the plant. The objective is to drive the error (difference between desired and actual heading) to zero in an optimal (critically damped) way (Fig. 8). The feedback loop, through the digital compass, is used to monitor the desired outcome and continuously update the calculated error. The 


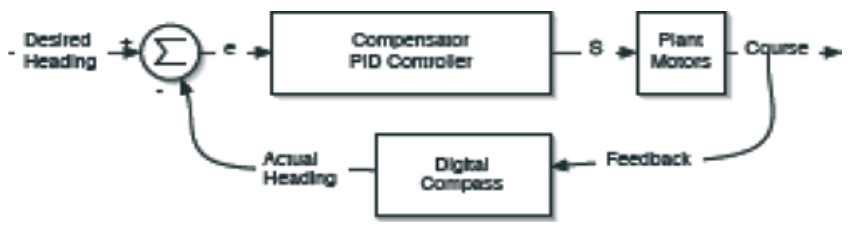

Figure 8: Functional control loop for heading.

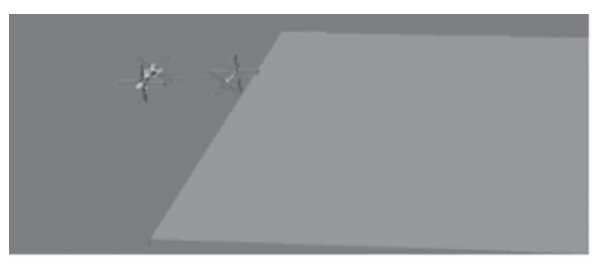

(a)

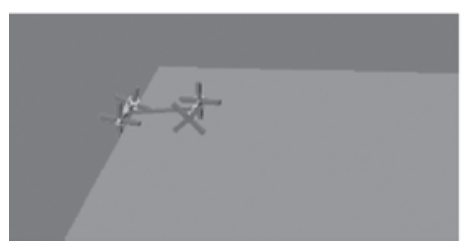

(c)

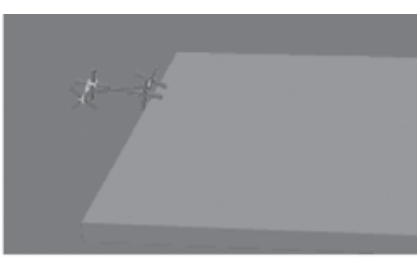

(b)

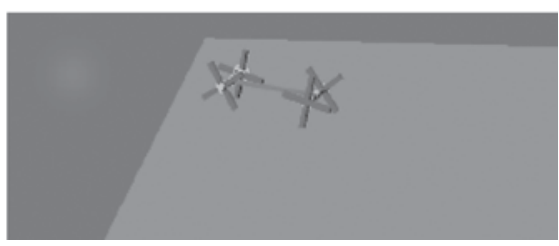

(d)

Figure 9: Simulation of insect-inspired robot and impact of passive compliance in locomotion.

proportional, integrative, and derivative gain coefficients were established through an in-field tuning process using a modified Ziegler-Nichols Method.

\subsection{Performance simulation}

Finally, we have constructed a simple simulation environment such that our beach robot may be tested dynamically in simulation prior to construction. The results of one such simulation run in this environment are shown in Fig. 9. In this simulation, a quadruped robot driven by four spoked wheel-legs approaches a plateau which it must climb. The height of the plateau in this simulation is exactly equal to the height of each wheel-leg (measured from the geometric center). The body of the robot was modeled as a rod attached to two revolute joints, each of which was connected to a shaft driving the wheel-legs. The weight of the rod was $10 \mathrm{~kg}$ representing the robot sensor payload, motors, and chassis. The weight of each shaft was $1 \mathrm{~kg}$, and the weight of each wheel-leg was $300 \mathrm{~g}$. As with the actual robot, each wheel-leg was also equipped with a compliant mechanism, in the form of a torsional spring with a constant of $0.2 \mathrm{~N} \mathrm{~m} / \mathrm{deg}$. The robot was also designed to run in a diagonal gait where each wheel-leg was moving in phase with the wheel-leg diagonally opposite itself. Each snapshot in Fig. 9 shows phases of the dynamic simulation. Figure 9A shows the robot just prior to reaching the vertical step to the plateau. Note that the robot has not reached the plateau, and is still moving in a diagonal gait. Figure $9 \mathrm{~B}$ shows the robot as it is beginning to 
climb the step. As can be seen from the figure, the front wheel-legs have reached the step and, due to the action of the compliant mechanisms, have passively shifted into phase with one-another to complete the climb. The two rear wheel-legs, however, are still out of phase. In Fig. 9C, the two front legs have returned to their out-of-phase position having surmounted the climb, while the two rear wheel-legs have now passively adjusted to be in phase as in order to effectively climb the obstacle. Finally, Fig. 9D shows the robot having completely surmounted the climb, passively returning to its diagonal gait. These results clearly demonstrate the capacity of insect-inspired design to passively adjust its gait to arduous terrain. This simulation testbed was used to dynamically examine the locomotive capability of our design to insure proper functionality for all manner of amphibious operations.

A two-dimensional (2D) dynamic simulation of the robot was also created using Working Model 2D 7.0. During climbing in the real robot, the left and right wheel-legs slide into phase with each other, due to the torsional compliance. This load sharing greatly improves roll stability [1]. This allowed us to simplify the 2-D model as a robot with only three wheel-legs. We also assumed that all three wheel-legs moved together with a constant velocity. We did not include the torque limit of the motor in the model. This allowed us to safely predict the maximum height of an obstacle the robot can overcome, given its dimensions and weight distribution. Given a robot weight of $21 \mathrm{~kg}$ and a center of gravity coincident with the center of geometry, a wheel leg radius of $19 \mathrm{~cm}$, and a coefficient of friction of 0.3 , the simulated robot was able to consistently climb an obstacle of $30 \mathrm{~cm}$ height.

Figure 10 shows the robot with all these features, including the new foot design, steering linkage, integrated autonomous GPS navigation hardware, and all sensor components.

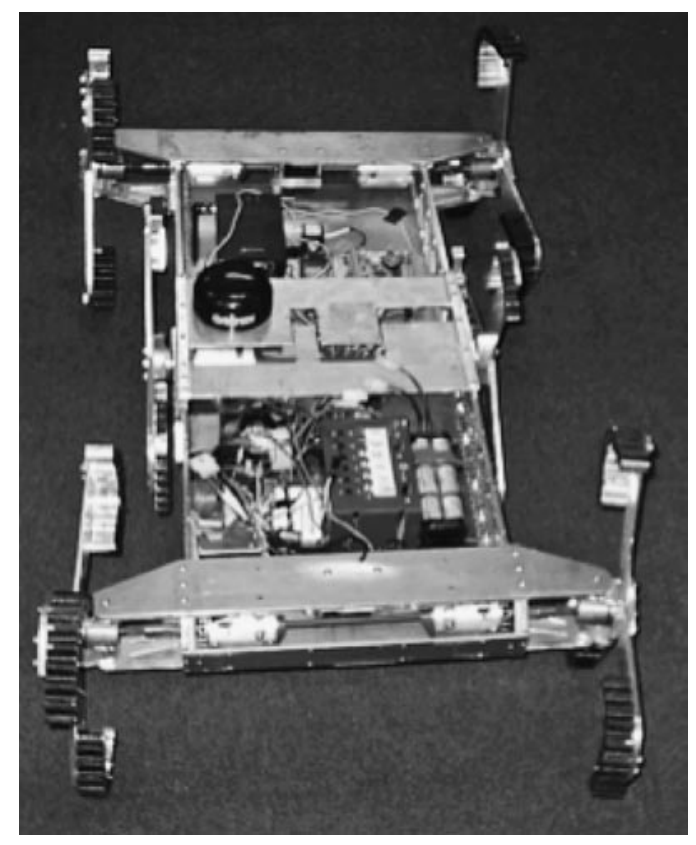

Figure 10: Beach robot with top enclosure removed to show mechanical and electrical components. 


\section{BEACH ROBOT PERFORMANCE}

\subsection{Locomotion}

In a series of field experiments, the beach robot was tested for mobility in loose soil, on man-made roads and stairs, and in hard-packed sand with a transition to soft, dry sand in $20^{\circ}$ to $30^{\circ}$ inclines. In the hard-packed sand and on man-made roads the robot, with minor exceptions, maintained the tripod gait. Passive compliance was observed in loose soil and the transition to soft sand without any loss of mobility or control. In contrast, wheeled robots were tested in this terrain and observed to 'bog down' in the exact same soft sand conditions. Inclines over approximately $30^{\circ}$ in soft sand proved challenging for the robot, yet mobility was maintained with some loss of speed due to slippage of the feet. Passive gait change was also observed more frequently over higher inclines. Figure 11 shows a series of snapshots of the robot crawling over loose soil on a very steep incline. Figure 12 shows a series of snapshots of the robot climbing a set of concrete stairs, where the action of the passively compliant joints may be observed in a manner similar to the simulation in Fig. 9. Figure 13 shows a snapshot of one experiment of the robot maintaining mobility under autonomous control over soft sand. Note the depth of the footprints left by the feet in the soft sand, indicating the actions of the feet and additional forces provided by the passive mechanisms of the robot. Again, the robot was able to maneuver through each of these substrates with no modification to its design or control subsystems. This adaptability is enabled through the insect-like integration of passive mechanics with the active control mechanisms in the robot.

The robot was able to climb full-height stairs $(19 \mathrm{~cm})$, both indoors and outdoors. The maximum height single obstacle it overcame was a $25-\mathrm{cm}$ shelf. More irregularly shaped objects such as boulders would sometimes pose a problem, as the body would get stuck on top of the obstacle with the legs to the sides. In most cases, the operator was able to get the robot untangled from the boulder using teleoperation. In the future, a body joint would greatly help it extricate itself from such situations. We observed that occasionally the front of the robot body would hit the vertical surface of the stair before the wheel leg could lift it over. Adding a hub to the wheel leg design should prevent this
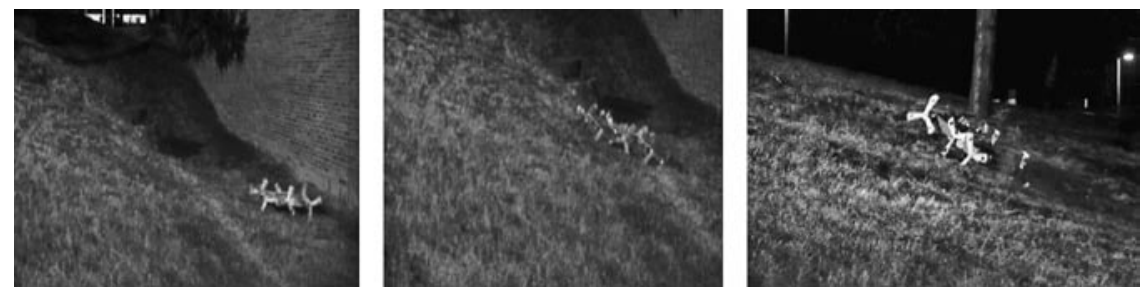

Figure 11: Snapshots from a mobility test of the robot in loose soil on a steep incline.
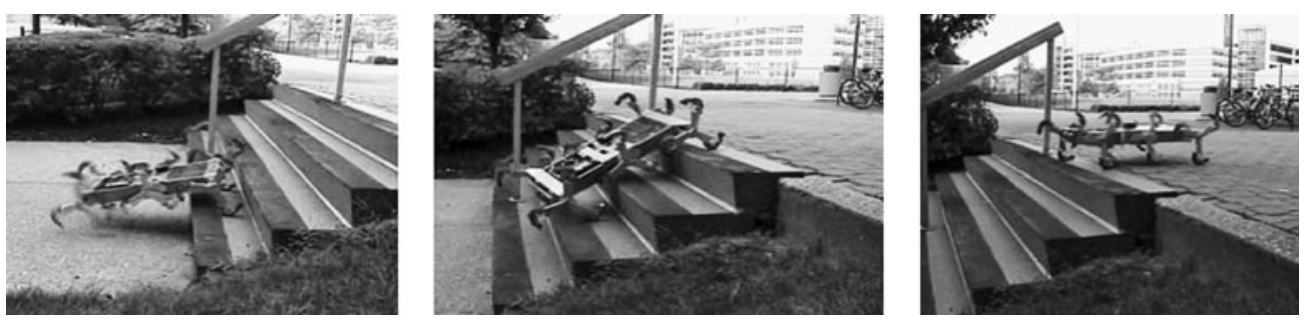

Figure 12: Snapshots from a mobility test of the robot climbing cement stairs. 


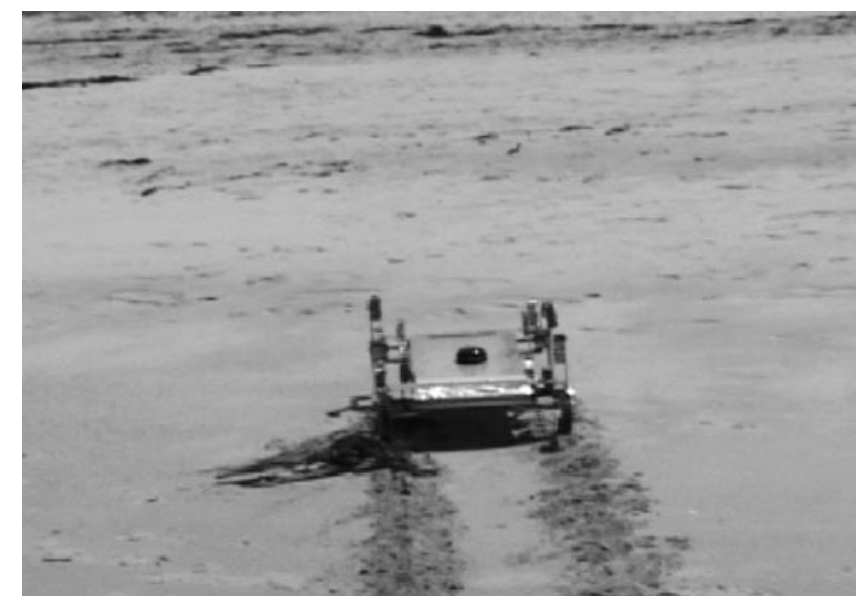

Figure 13: Robot crawling over soft sand on Monterey beach.

from happening in the future. We also observed that the profile of the wheel leg spoke was such that the contact point with the stair was always farthest from the hub. This means that the motor has to apply maximum torque at all times. A small change in the profile of the spoke to incorporate a gradual curve would minimize the time the motor is applying a maximum torque.

\subsection{Autonomous control}

Autonomous waypoint navigation was demonstrated in a facsimile demining mission on the beach in the non-submersed surf-zone region. In the facsimile mission (Fig. 14), a series of waypoints were given to the robot (representing possible mines). The robot was to navigate autonomously over any obstacles or change in substrate to each waypoint, circle the waypoint to inspect the possible threat, and move to the next location. The robot recovered from all terrain perturbations in the field environment while in transit to multiple waypoints. Navigation was based on GPS data. Figure 14A-F shows progressive snapshots of the robot walking autonomously on the beach over both hard and soft sand to reach a series of waypoints (designated by flags in the snapshots).

\section{DESIGN OF AMPHIBIOUS SURF-ZONE ROBOT}

Based on the results from our beach robot, we designed an amphibious Surf-Zone Whegs ${ }^{\mathrm{TM}}$ robot for movement on the sea floor, swimming and running on the beach. Similar to typical Whegs robots, it has a single motor that drives all six of its wheel-legs through torsional compliant devices. Some of the design differences that enable amphibious locomotion are described below.

\subsection{Mechanical design}

This platform will have the ability to swim or walk in the transit towards the beach. We are in the process of developing a new multi-modal locomotion mechanism combining aquatic propeller propulsion with terrestrial locomotion capacity provided by Whegs ${ }^{\mathrm{TM}}$. Propeller wheel-legs (Fig. 15, left) will propel the next generation robot in both crawling and swimming modes.

In subsequent work, we will develop a lighter, carbon fiber or Kevlar, water-tight vehicle that can be tested in the water and in transit to the beach to reproduce these results while transitioning from aquatic to terrestrial environs. The robot will use two different steering methods for walking and swimming. 


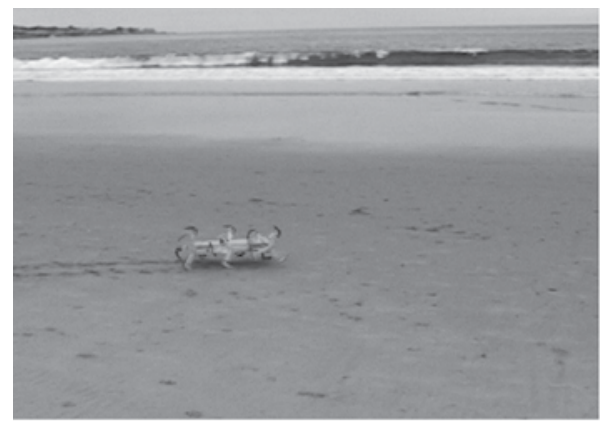

(a)

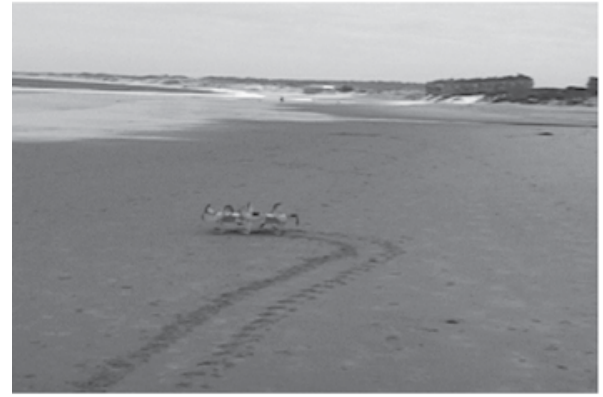

(c)

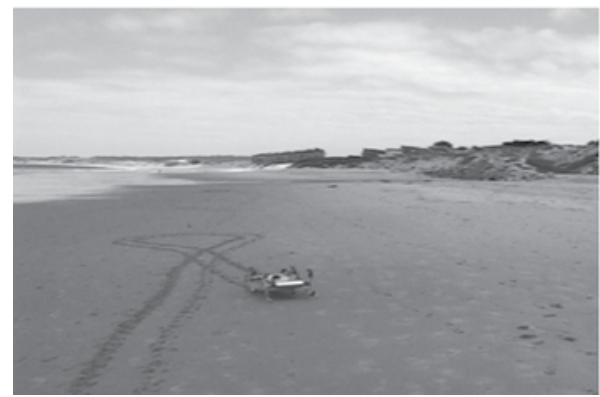

(e)

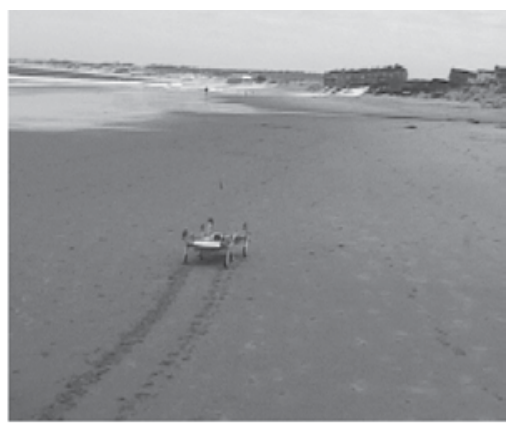

(b)

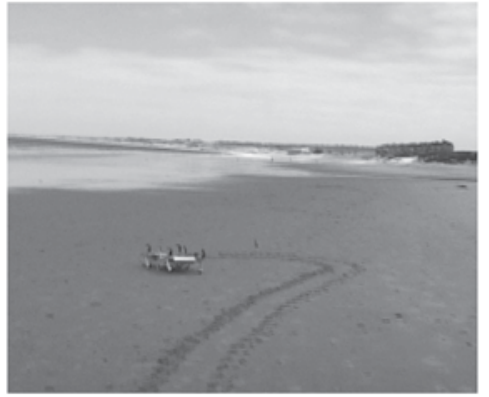

(d)

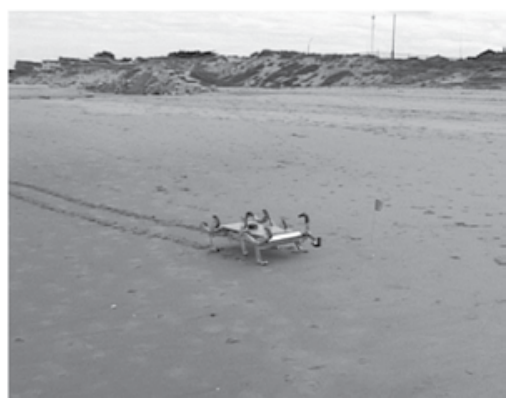

(f)

Figure 14: (A-F): Progressive snapshots of the robot walking autonomously on a beach to reach waypoints.

5.1.1 Component layout

The robot is comprised of two body segments that are nearly symmetrical (Fig. 16). The front body segment will leave as much room as possible for sensors and related electronics. It will only contain the drive motor, steering servos, and a speed controller. In previous Whegs ${ }^{\mathrm{TM}}$ robots, the compliant mechanisms were contained inside the frame. However, there is no need to waterproof these mechanisms, so they have been moved outside the sealed frame to save space. All drive chains run along the sides to prevent dividing up the usable space. The front and rear bulkheads of the robot will be rounded to give good hydrodynamic characteristics and to allow it to push up and over irregularly shaped obstacles. Windows can be easily added to the bulkhead to allow video cameras to be stored inside the front body segment. 

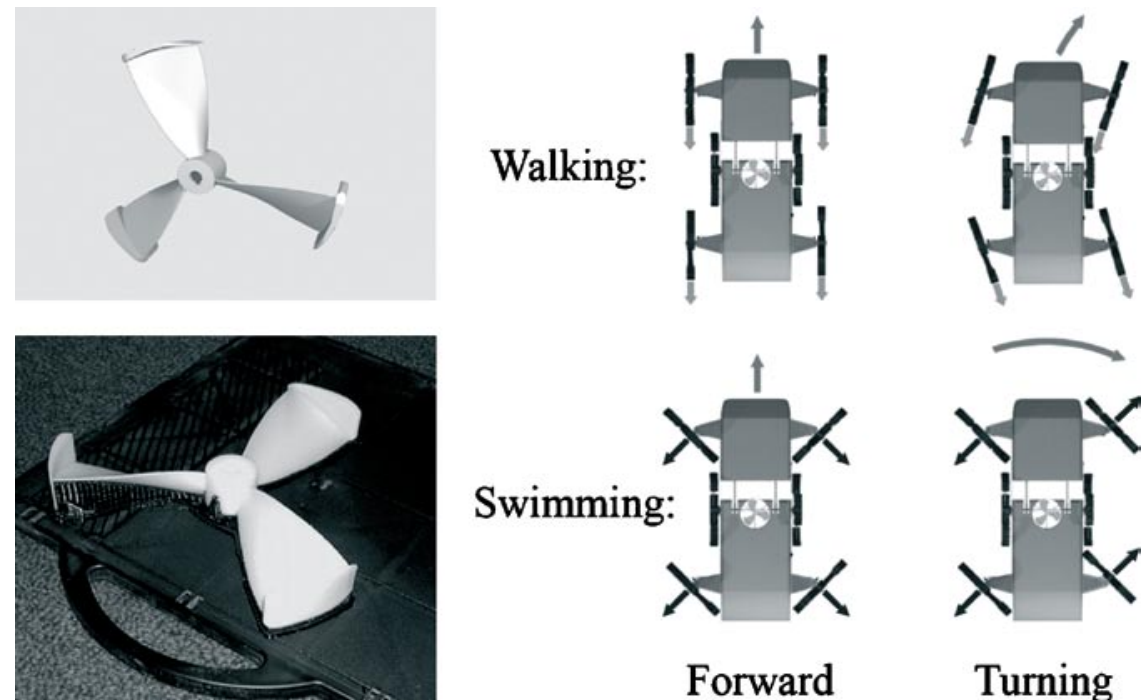

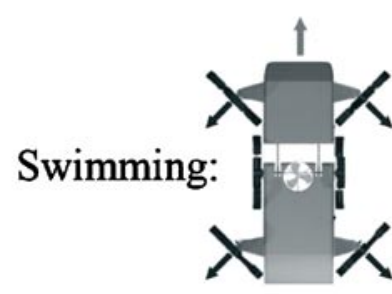

Forward

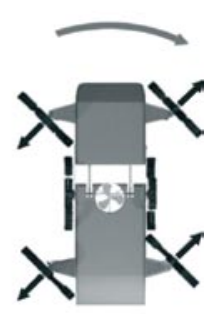

Turning

Figure 15: The black arrows show thrust created by the propellers in swimming mode (bottom), while the gray arrows show the force acting on the ground in walking mode (top). The dashed arrows indicate the resultant direction of the robot.

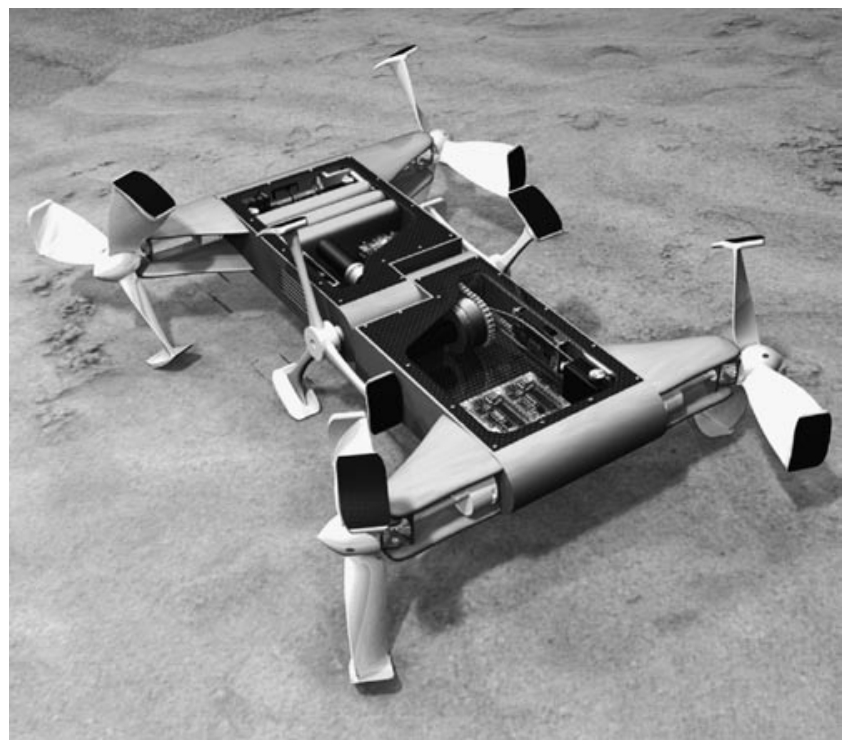

Figure 16: Cutaway rendering of component layout. The front compartment contains the drive motor, speed controller, and steering servos. The remaining space is available for a control board (shown), compass, GPS, and other sensors (not shown). The rear compartment is shown with the body joint motor, speed controller, batteries, and servo controllers. The batteries can be placed anywhere in the robot for optimal balance. 


\subsubsection{Sealed body}

The surf-zone robot is completely encased, keeping dirt as well as water out. Both body segments are constructed from a ring of aluminum side panels with carbon fiber tops and bottoms. Each set of side panels is sealed to itself using silicone gasket, while the carbon fiber panels are sealed to the side panels using rubber gaskets. This will allow the robot to be easily serviced by removing the carbon fiber panels without breaking the seals between the side panels.

Rotary axles must also penetrate the body of the robot in seven places, one for each wheel-leg and the body joint. Several rotary shaft seals are in consideration for this task. Our first prototype will test urethane U-cup seals that use the outer ambient pressure to keep the seal against the shaft and housing. Other possibilities under consideration include mechanical seals or multiple o-rings.

The weight of the current robotic platform is $18 \mathrm{~kg}$. For initial operation under water, the robot will function only on the sea floor, so we want it to be slightly negatively buoyant. The density of water is $1000 \mathrm{~kg} / \mathrm{m}^{3}$, so the robot needs to displace $0.02 \mathrm{~m}^{3}$. The sealed compartment of the robot will be roughly $0.10 \mathrm{~m} \times 0.25 \mathrm{~m} \times 0.75 \mathrm{~m}=0.019 \mathrm{~m}^{3}$, making it nearly neutrally buoyant. At this dimension, a small amount of diving weights can be added during initial operation and removed for swimming. Future work could also investigate adjustable ballast tanks to allow the robot to transition from the sea floor to swimming when desired.

\subsubsection{Body joint}

Connecting the two body segments with an insect-like body joint presented many design challenges. Torque must be transmitted from the front motor to the rear wheel-legs. Power and communication lines must be passed between the two body segments. The body joint must be actuated with a motor and any linkage must be water and dirt tight. A pair of coaxial shafts is used: the outer one is rigidly attached to the front body segment, allowing a motor in the rear body segment to actuate the body joint; the inner shaft is the middle wheel-leg drive shaft, which also passes torque to the rear of the robot. The outer shaft is $6.35 \mathrm{~cm}$ in diameter, large enough to run several electrical lines through. By keeping all connections axial in nature, a standard rotary shaft seal around the outer shaft can be used to keep water and dust out (Fig. 17).

A cockroach-like body joint will allow the robot to climb larger objects by giving the front wheellegs higher reach and by preventing high centering. However, the rugged surf-zone environment precluded several designs that did not survive field testing. The first version body joint used a large backdrivable servo, which allowed the motor to absorb some of the shock of impact, but constant current draw quickly drained the batteries and affordable commercial servos were not rugged enough for repeated impacts. To remedy this, we considered a non-backdrivable worm gear. However, the teeth of the gear sheared off under impact loading. It was clear that a new body joint actuator needed to be developed. The solution in surf-zone robot is a compliant, non-backdrivable body joint. A motor with a transmission is connected to a worm that drives the worm gear. In this modified design, the worm can slide axially but not radially on the shaft that is cushioned on both sides by Belleville springs (Fig. 18). A large axial bolt holds the bearings in place and tensions the Bellville springs. When the front wheel-legs impact an obstacle, the front body segment rotates up and back, rotating the driven worm gear, which pushes the driving worm in a fashion similar to a rack and pinion, allowing the Belleville springs to cushion the blow. Regardless of the passive state of the body joint, the motor can actuate the body joint in either direction.

This design essentially puts a spring in series with an actuator and is similar to the recently introduced series elastic actuator [15]. Unlike a series elastic actuator, which is force controlled, this joint is partially passive, acting like a car suspension, independent of actuation. This non-backdrivable design is also inherently rotary, eliminating the need for cables. 


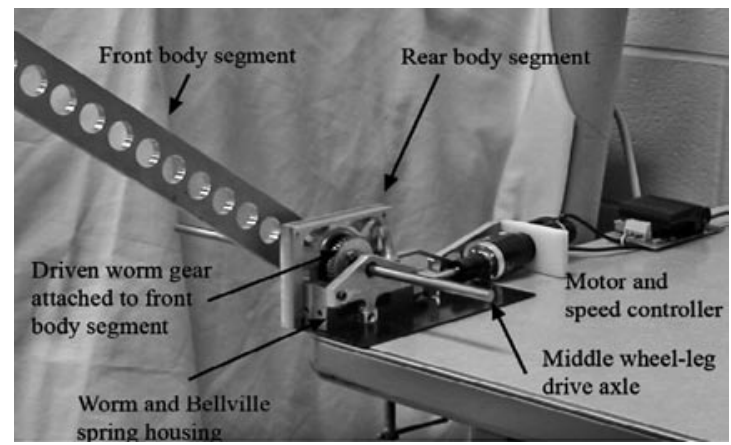

Figure 17: Body flexion joint that can be water tight, compliant, actuated, and allows cables to pass between the two body segments.

(a)

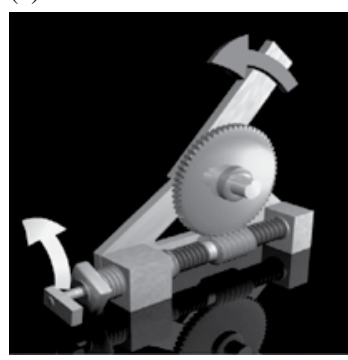

(b)

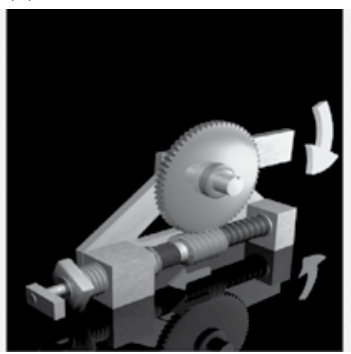

(c)

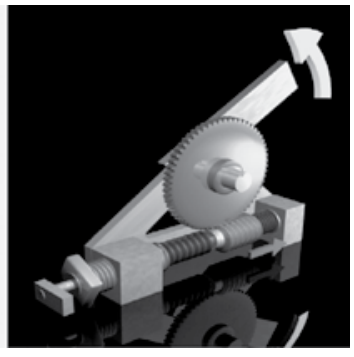

Figure 18: A simplified diagram of the body joint demonstrates its multiple functions. When the worm is driven by a motor (white arrow in A), the body joint changes position similar to a standard worm gear (gray arrow in A). When a force is applied to the driven gear (white arrows in B and $\mathrm{C}$ ), the worm slides axially on the drive shaft, where Bellville springs cushion the blow (gray arrows in B and C).For the full sequence see: http://biorobots.case.edu for videos.

This design also allows the passive stiffness of the body joint to be independently tuned in the clockwise and counter-clockwise directions by changing the number and stiffness of the Belleville springs on either side of the worm. When run autonomously, it may be advantageous to have a very low stiffness body joint that works entirely passively to overcome obstacles. When in radio control mode, the body joint stiffness can be higher to allow more responsive user control.

Figure 19 shows the first prototype body joint that was built and tested for the surf-zone robot. Using a $20 \mathrm{~W}$ motor and a 23:1 gear reduction, our calculations predicted a stall torque of $83.5 \mathrm{~N} \mathrm{~m}$ and a slew rate of $56 \mathrm{deg} / \mathrm{s}$ under a torque of $14.7 \mathrm{~N} \mathrm{~m}$. Experimentally, we were not able to find the stall torque because one of the two axial ball bearings that supported the Bellville springs failed while testing a load of $26.4 \mathrm{~N} \mathrm{~m}$. Resonant vibrations were observed, which may have contributed to the failure. Future work will include rebuilding the body joint with bushings instead of bearings and finding the stall torque and slew rate in order to find the real efficiency of the system.

With the exception of the bearing failure, the body joint performed as expected. The passive compliant range of motion of the Bellville springs was $\pm 12^{\circ}$, slightly less when tested with stiffer springs. The body joint could rotate over a full $360^{\circ}$ without variations in speed, and rotate continuously. Shaking the lever arm in all directions did not appear to affect the performance. Changing the Bellville springs was relatively easy and changed the stiffness of the lever arm as expected. 

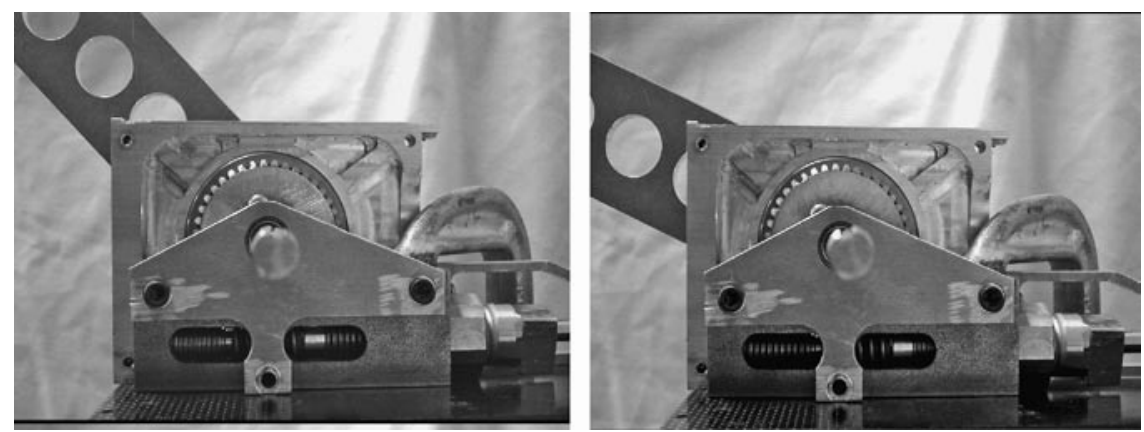

Figure 19: Demonstration of the passive compliance of the body flexion joint. In the top photo, the joint is loaded counterclockwise under $20 \mathrm{~N} \mathrm{~m}$. In the bottom photo, the joint is loaded clockwise under $14 \mathrm{~N} \mathrm{~m}$. The independently tuned Bellville springs on both sides of the worm give it this non-backdrivable compliance.

\section{CONCLUSIONS AND FUTURE WORK}

The objective of this research was to develop a prototype beach robot capable of navigating complex terrain and changing substrates seamlessly and autonomously. A secondary goal was to determine if the insect-inspired Whegs ${ }^{\mathrm{TM}}$ platform was robust enough and mobile enough to operate in this environment to justify future work in this area. A third goal was to demonstrate how simplified, implementable robust control can enable complex locomotion over varying terrain through synergistic interaction with passive mechanisms in mechanical design. It is our opinion that our data confirm all these objectives. Furthermore, we have also leveraged these accomplishments to design an amphibious surf-zone robot for movement on the sea floor, swimming and running on the beach.

We report the successful design, construction, and implementation of communication and autonomous control systems on a robot capable of navigating the challenging terrain of the non-submersed surf-zone region. The robot benefits from insect-inspired mechanisms of locomotion for movement over various and different terrains. The robot's mechanics are an integrated and essential part of its control system. It does not have (or need) sensors and control circuits to actively change its gait. Instead, its mechanics cause it to passively adapt its gait appropriately to very different terrains. Therefore, its motor control circuits are only needed to control speed and heading of the robot much like that of a wheeled vehicle. Its navigational system is a higher-level circuit that communicates desired speed and heading to the local control system. Therefore, the beach robot has a control system with the simplicity of a wheeled vehicle but with the mobility of a legged vehicle.

\section{ACKNOWLEDGMENTS}

The authors gratefully acknowledge the generous support of the Temasek Defence Systems Institute, National University of Singapore, under the program 'Autonomous Amphibious Robots for Surf Zone Operations' (reference: TDSI/06-006/1A). We also cite the contribution of the many students at the US Naval Postgraduate School and Case Western Reserve University who have contributed to this project, Captain (ret.) Jeffery E. Kline (USN) for mission definition and utility insights, and the continued support of Dr. David Olwell and the Department of Systems Engineering at the US Naval Postgraduate School. We also cite the laboratory of Dr. Roy Ritzmann in the Departments of Biology and Neuroscience at Case Western Reserve University for insights and experiments into cockroach locomotion. Partial support was also provided by the Dayton Area Graduate Studies Institute (DAGSI) and BioRobots Ltd. 


\section{REFERENCES}

[1] Boxerbaum, A.S., Werk, P., Quinn, R.D. \& Vaidyanathan, R., Design of an autonomous amphibious robot for surf zone operation: part I, mechanical design for multi-mode mobility. IEEE/ASME International Conference on Advanced Intelligent Mechatronics, Monterey, CA, USA, 2005.

[2] Bernstein, C., Connolly, M., Gavrilash, M., Kucik, D. \& Threatt, S., Demonstration of surfzone crawlers: results from AUV fest 01, Surf Zone Crawler Group, Naval Surface Warfare Center, Panama City, FL, USA, 2001.

[3] iRobot Corporation, Ariel Robot, http://www.irobot.com/about/history.cfm, 2005.

[4] Prahacs, C., Saunders, A., Smith, M., McMordie, D. \& Buehler, M., Towards legged amphibious mobile robotics. The Inaugural Canadian Design Engineering Network (CDEN) Design Conference, Montreal, Canada, 2004.

[5] Crespi, A., Badertscher, A., Guignard, A. \& Ijspeert, A.J., Amphibot I: an amphibious snake-like robot. Robotics and Autonomous Systems, 50, pp. 163-175, 2005. doi:10.1016/j. robot.2004.09.015

[6] Georgidas, C., German, A., Hogue, A., Liu, H., Prahacs, C., Ripsman, A., Sim, R., Torres, L.-A., Zhang, P., Buehler, M., Dudek, G., Jenkin, M. \& Milios, E., AQUA: an aquatic walking robot, Proc. Unmanned Underwater Vehicle Systems (UUVS), Southampton, UK, 2004.

[7] Saranli, U., Buehler, M. \& Koditschek D., RHex a simple and highly mobile hexapod robot. International Journal of Robotics Research (IJRR), 20, pp. 616-631, 2001. doi:10.1177/02783640122067570

[8] Quinn, R.D., Kingsley, D.A., Offi, J.T. \& Ritzmann, R.E., Improved mobility through abstracted biological principles. IEEE Int. Conf. on Intelligent Robots and Systems (IROS), Lausanne, SZ, 2002.

[9] Harkins, R., Ward, J., Vaidyanathan, R., Boxerbaum, A.S. \& Quinn, R.D., Design of an autonomous amphibious robot for surfzone operations: part II. IEEE/ASME International Conference on Advanced Intelligent Mechatronics, Monterey, CA, USA, 2005.

[10] Quinn, R.D., Nelson, G.M., Ritzmann, R.E., Bachmann, R.J., Kingsley, D.A., Offi, J.T., \& Allen, T.J., Parallel strategies for implementing biological principles into mobile robots. International Journal of Robotics Research (IJRR), 22, pp. 169-186, 2003. doi:10.1177/0278364903022003003

[11] Ritzmann, R.E., Rice, C.M., Pollack, A.J., Ridgel, A.L., Kingsley, D.A. \& Quinn, R.D., Roles of descending control in locomotion through complex terrain. Congress of Neuroethology, $\mathbf{6}$, p. 234, 2001.

[12] Watson, J., Ritzmann, R., Zill, S. \& Pollack, A., Control of obstacle climbing in the cockroach, Blaberus discoidalis. I. Kinematics. Journal of Comparative Physiology, 188, p. 3953, 2002. doi:10.1007/s00359-002-0277-y

[13] Ritzmann, R.E., Quinn, R.D. \& Fischer, M.S., Convergent evolution and locomotion through complex terrain by insects, vertebrates and robots. Arthropod Struc. Dev., 33, pp. 361-379, 2004. doi:10.1016/j.asd.2004.05.001

[14] Allen, T., Quinn, R.D., Bachmann, R.J. \& Ritzmann, R.E., Abstracted biological principles applied with reduced actuation improve mobility of legged vehicles. IEEE International Conference on Intelligent Robots and Systems (IROS), Las Vegas, Nevada, 2003.

[15] Robinson, D.W., Pratt, J.E., Paluska, D.J. \& Pratt, G.A., Series elastic actuator development for a biomimetic robot. IEEE/ASME International Conference on Advance Intelligent Mechatronics, Atlanta, GA, USA, 1999. 